\title{
Provider Deferred Decisions on Hemoglobin A1c Results: A Report from the Colorado Research Network (CaReNet) and the High Plains Research Network (HPRN)
}

\author{
Bennett Parnes, MD, Linda Niebauer, Sherry Holcomb, Miriam Dickinson, PhD, \\ Jack Westfall, MD, Becky VanVorst, and Wilson Pace, MD
}

Background: Hemoglobin A1c (HbA1c) results are generally reviewed several days after office visits. The clinical decisions on elevated HbA1cs may be complex and are rarely urgent. Providers may elect to defer the decision or its implementation to a future clinical encounter.

Objective: To determine the occurrence rate, predictors, and eventual decision outcomes for HbA1c deferred decisions.

Design: Provider questionnaire completed when HbA1c results from type 2 diabetes patients were reviewed, followed by a chart review on deferred cases 6 months later.

Participants: Providers at 19 Colorado primary care clinics.

Measurements: For HbA1c $\geq 7 \%$, whether the decision or its implementation was deferred. In deferred cases, whether a clinical decision was eventually made.

Results: Of the $311 \mathrm{HbA1cs} \geq 7 \%, 31(10.0 \%)$ had deferred decisions. In multivariate analysis, deferred decisions were more likely in African Americans (odds ratio [OR] 4.91, 95\% CI 1.81, 13.3) and less likely when the patient's usual provider reviewed the HbA1c $(0 \mathrm{R} 0.40,95 \% \mathrm{CI} 0.18,0.90)$. In the chart review, for deferred cases $(n=18)$, a clinical decision was made in 14 cases, usually at the next clinical encounter. In 4 cases, the HbA1c was never addressed.

Conclusion: Deferred decisions on HbA1c results are infrequent, and usually the HbA1c is eventually addressed. (J Am Board Fam Med 2006;19:20-3.)

Intensive glycemic control, as measured by serum hemoglobin A1c (HbA1c) levels, ${ }^{1}$ has been demonstrated in randomized trials to reduce diabetic complications, especially microvascular disease. ${ }^{2,3,4}$ The American Diabetes Association (ADA) HbA1c guideline is the most widely disseminated in the United States. ${ }^{5}$ At the time of this study, the ADA-

Submitted 9 November 2004; revised 13 June 2005; accepted 13 June 2005.

From the Department of Family Medicine, University of Colorado School of Medicine, Health Sciences Center, Aurora, CO.

Funding: This study was supported by the American Academy of Family Physicians Foundation.

This paper was presented at the North American Primary Care Research Group's Annual Meeting in San Diego, CA, October 2003.

Conflict of interest: none declared.

Corresponding author: Bennett Parnes, Department of Family Medicine, University of Colorado School of Medicine, Health Sciences Center, 12474 E. 19th Avenue, Building 402, PO Box 6508, Campus Box F496, Aurora, CO 80045-0508 (E-mail: bennett.parnes@uchsc.edu). recommended target for $\mathrm{HbA1}$ c was $\leq 7 \%$. Clinical intervention was recommended for $\mathrm{HbAlc} \geq 8 \%$ and was to be considered for HbA1c between $7 \%$ and $8 \%$.

A key factor in achieving glycemic control is the clinician's decision concerning the HbA1c result. In the typical outpatient setting, HbA1c results are obtained by venipuncture at a clinic encounter and reviewed by the provider 1 to 7 days later. In a previous report, we found that primary care providers took action on elevated HbAlc most of the time, and we described provider-reported reasons when no action was taken. ${ }^{6}$

The clinical management of an elevated HbA1c level can involve many factors, including assessment of patient medication and lifestyle adherence, the frequency of hypoglycemic episodes, formulary concerns, the issues in initiating insulin therapy, etc. These considerations may require a lengthy telephone call to the patient or another clinic visit. 
This contrasts with clinical decisions on many other abnormal lab results that are used to monitor chronic disease. For example, in a patient who has hypothyroidism and is on thyroid replacement, an abnormal thyroid-stimulating hormone (TSH) typically can be managed with a short phone call or letter to the patient, because only a simple dosage adjustment is required.

The decision to take action on an abnormal HbA1c result is rarely urgent. One quality assessment tool stated that up to 3 months is reasonable for changing management due to an elevated HbA1c. ${ }^{7}$ Therefore, given the multiple factors that may be involved in HbA1c management decisions, a reasonable option could be to defer the decision or implementation of the decision until the next clinical encounter. We are aware of no previous reports that have investigated deferred clinical decisions on HbA1c or any other lab results.

The purpose of this report is to describe deferred clinical decisions on HbA1c lab results, including the frequency of occurrence, the factors associated with the deferred decisions, and what ultimately happens in these deferred cases.

\section{Methods}

A complete description of the survey and data collection methods can be found in a previous report. ${ }^{6}$ In brief, the participants in this study consisted of 88 anonymous providers from 19 primary care clinics in 2 practice-based research networks-the Colorado Research Network (CaReNet) and the High Plains Research Network (HPRN). CaReNet is a largely urban network of practices with a high percentage of underserved patients. HPRN clinics are located in rural and frontier northeast Colorado.

Each provider completed a short questionnaire after reviewing every HbAlc result from adult nonpregnant patients with type 2 diabetes. The questionnaires were administered during a 3- to 6-week period at each clinic between December 2001 and August 2002. For each HbA1c result, providers reported that they either: (1) took action (eg, recommended lifestyle or medication change), (2) did not take action, or (3) deferred the clinical decision or the action. Deferred decision or action was defined as when the provider delayed a decision about an action until the next clinical encounter or when the provider decided to take action but did not plan to implement the intervention until the patient's next clinical encounter.
In cases with an $\mathrm{HbA1c}$ value $\geq 7 \%$, we conducted univariate and multivariate analyses to identify predictors for deferred cases compared with decisions made and implemented at the time of the initial laboratory result review.

In deferred cases with an $\mathrm{HbA1c}$ result $\geq 7 \%$, we performed a retrospective chart audit on all available charts 6 months after the HbA1c was obtained. For each case, we determined whether the HbA1c was eventually addressed, and if so, whether or not action was taken.

This study was approved by the Institutional Review Boards overseeing research in the participating clinics.

\section{Results}

Of 483 total questionnaires completed, 311 had an HbA1c value $\geq 7 \%$. Of these 311 cases, providers took action in 198 cases $(63.7 \%)$, took no action in $82(26.4 \%)$, and deferred the clinical decision or action in $31(10.0 \%)$. The mean HbAlc on deferred cases was $8.9 \%$ compared with $9.2 \%$ in nondeferred cases.

Similar findings were obtained in both the univariate and multivariate analyses that compared the 31 deferred cases to the 280 non-deferred cases. The univariate results are presented in Figure 1. African American patients were more likely to be in the deferred group $(\mathrm{n}=7 ; 22.6 \%$ of the deferred cases) compared with the non-deferred group ( $\mathrm{n}=$

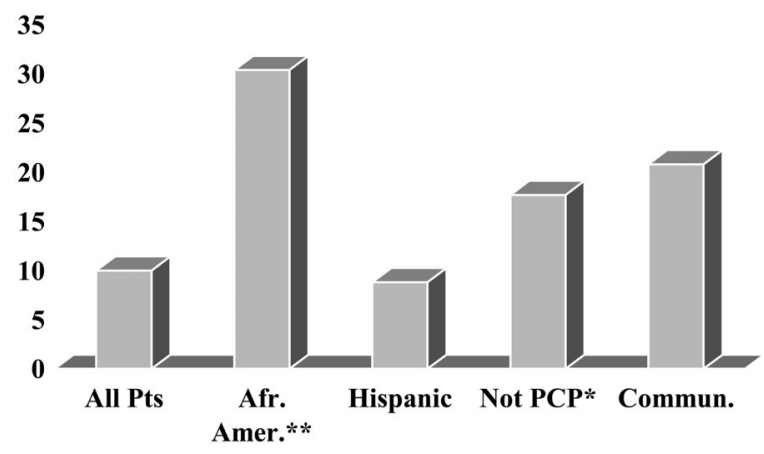

Figure 1. Deferred rate by characteristic univariate analysis. $*, \boldsymbol{P}<.05 ; * *, \boldsymbol{P}<.01 ; \chi 2$ with continuity correction; not significant for gender, insurance, A1c level, number of glycemic medicines, number of comorbidities. Afr. Amer., African American; PCP, primary care provider; Commun., there was a communication problem between the provider and patient. 


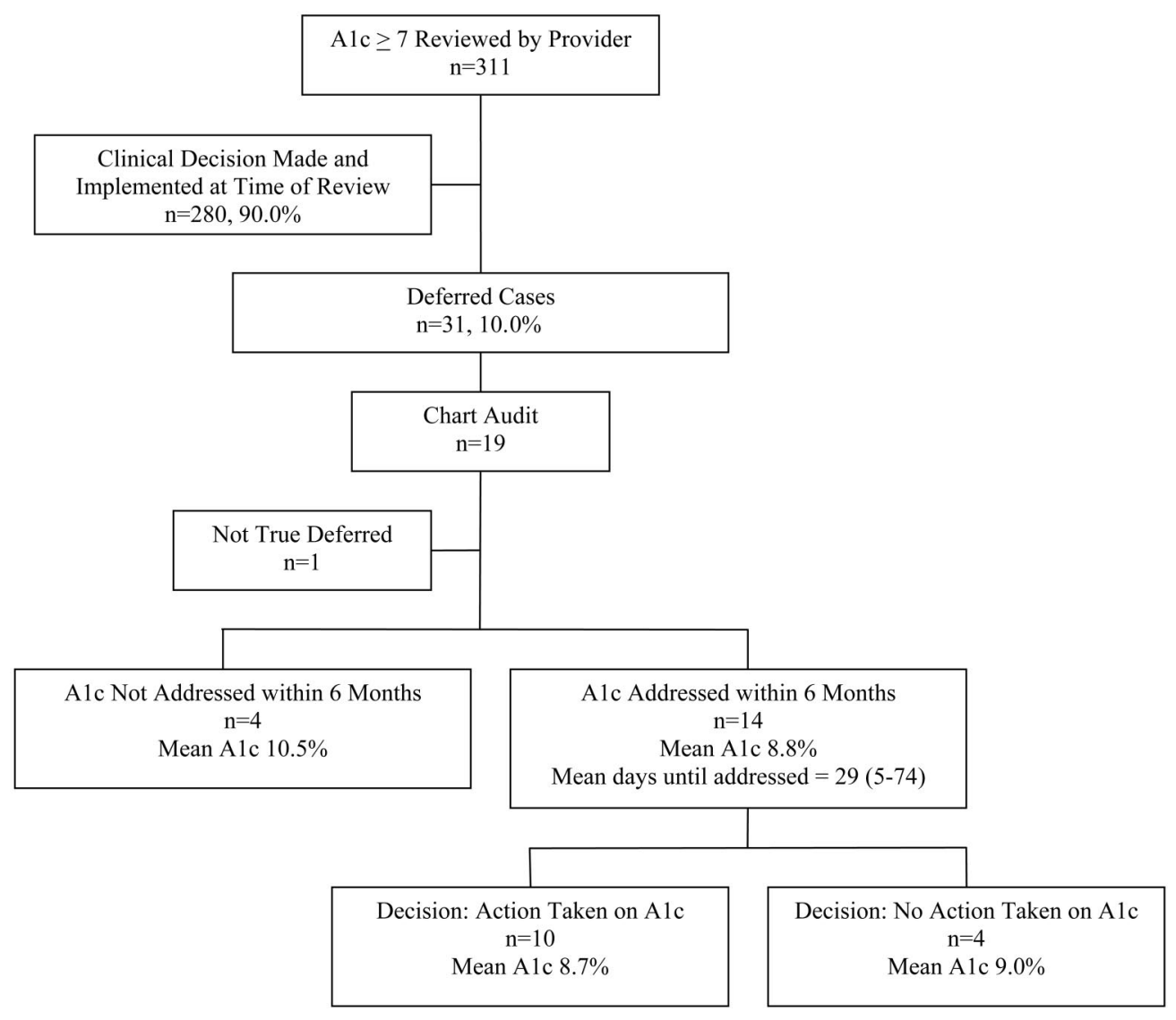

Figure 2. Outcomes for deferred cases.

16; $5.7 \%$ of the non-deferred cases), and patients who were not seen by their regular provider were more likely to be in the deferred group $(n=11$; $35.5 \%$ of the deferred cases) compared with the non-deferred group ( $\mathrm{n}=51 ; 18.2 \%$ of non-deferred cases). In the logistic regression model, being an African American patient continued to be a significant predictor for being in the deferred group (OR 4.91, 95\% CI 1.81, 13.3; $P=.003$ ), and being a patient seen by his or her regular provider remained a significant predictor that the decision would not be deferred (OR 0.40, 95\% CI 0.18, 0.90; $P=.028)$. No other patient characteristics were statistically significant in the multivariate model.

We were able to perform a chart review on 19 of the 31 deferred cases; in the other 12 cases, the identifying link to the chart was lost. We did not find any significant differences in demographics, insurance, or whether the patient was seen by his or her regular provider, between the 12 missing cases and the 19 that had a chart review.

In the chart review, one of the 19 cases was misclassified as deferred (on chart review, it was determined that a decision was made at the time of the original laboratory review). Of the remaining 18 cases, 4 HbAlc results (22\%) were never addressed. Of the 14 that were addressed, action was taken on 10 (71\%) (Figure 2).

\section{Discussion}

In this investigation of deferred clinical decisions on elevated HbAlc results in primary care, we found that deferred decision making is uncommon ( $10 \%$ of cases), and in most of these cases (78\%), the elevated HbA1c is eventually addressed. The action rate on deferred cases in which a clinical decision is eventually made $(71.4 \%)$ was similar to the action rate $(70.7 \%)$ when the clinical decision was made at the time of initial laboratory review.

It is intuitively clear why deferred cases are less likely when the patient's regular provider reviews the results. Other providers less familiar with the patient are less likely to change therapy. Our finding of a greater likelihood of deferred decisions for African American patients is more difficult to un- 
derstand. It is interesting that in our previous report, African American patients were less likely to have action taken on elevated $\mathrm{HbA} 1 \mathrm{c}$ results, ${ }^{6}$ and others have found racial disparities in diabetes care. ${ }^{8-10}$ However, it is less clear why disparities would be found in deferring a clinical decision. We did not find any disparities for Hispanic patients.

Deferred HbA1c decisions are clinically important because they may expose patients to prolonged periods with inadequate glycemic control, particularly if there is a long interval until the result is addressed or in cases in which the result is never addressed. However, in this study, most deferred decisions were eventually addressed, and the time interval until the clinical decision was implemented was relatively short (mean 29 days). Point-of-service HbA1c testing, which has been demonstrated to improve glycemic control in primary care ${ }^{11}$ but is not widely used, could eliminate most deferred decisions.

There are several important limitations in this study. First, there may be a Hawthorne effect. ${ }^{12}$ Providers may have been more likely to make a decision at the time of the initial HbA1c review because they were completing a questionnaire about their decision, rather than deferring their decision if there had not been a questionnaire. Therefore, the true deferred rate may be higher. Second, the number of deferred cases is relatively small, ${ }^{31}$ and African American patients are a relatively small minority group in these 2 practicebased research networks. Our findings would be best confirmed in a larger sample with a greater proportion of African Americans. Third, there may be provider or practice level effects, but due to sample size concerns, a multilevel analysis was not performed. Fourth, we were unable to perform the chart review on 12 of 31 cases. Even though we did not find significant differences between the 12 cases that were not reviewed and the 19 cases that were reviewed, the data on the eventual outcome in deferred cases should be interpreted with caution. Finally, we did not collect any information on why decisions were deferred, which may have included patient or provider factors.

Deferred clinical decisions on laboratory results have not been previously characterized in the medical literature. In this study, we found that deferred decisions on HbA1c results are relatively uncom- mon, and most of these are eventually addressed by providers in a timely fashion.

We thank the practices in CaReNet and HPRN that participated in this study, and also Elizabeth Staton and Stephanie Mitchell for assistance with the manuscript.

\section{References}

1. American Diabetes Association. Tests of glycemia in diabetes (Position Statement). Diabetes Care 2003;26 (Suppl. 1):S106-8.

2. UK Prospective Diabetes Study Group. Intensive blood-glucose control with sulphonylureas or insulin compared with conventional treatment and risk of complications in patients with type 2 diabetes (UKPDS 33). Lancet 1998;352:837-53.

3. Levin SR, Coburn JW, Abraira C, et al. Effect of intensive glycemic control on microalbuminuria in type 2 diabetes: Veterans Affairs Cooperative Study on Glycemic Control and Complications in Type 2 Diabetes Feasibility Trial Investigators. Diabetes Care 2000;23:1478-85.

4. Ohkubo Y, Kishikawa H, Araki E, et al. Intensive insulin therapy prevents the progression of diabetic microvascular complications in Japanese patients with non-insulin-dependent diabetes mellitus: a randomized prospective 6-year study. Diabetes Res Clin Pract 1995;28:103-17.

5. American Diabetes Association. Standards of medical care for patients with diabetes mellitus (Position Statement). Diabetes Care 2003;26(Suppl. 1):S3350.

6. Parnes BL, Main DS, Dickinson LM, et al. Clinical decisions regarding $\mathrm{HbA1c}$ results in primary care: a report from CaReNet and HPRN. Diabetes Care 2004;27:13-6.

7. Wenger NS Solomon DH, Roth CP, et al. The quality of medical care provided to vulnerable community-dwelling older patients. Ann Intern Med 2003;139:740-7.

8. Harris MI, Eastman RC, Cowie CC, Flegal KM, Eberhardt MS. Racial and ethnic differences in glycemic control of adults with type 2 diabetes. Diabetes Care 1999;22:403-8.

9. Cowie C, Harris MI. Ambulatory medical care for non-Hispanic whites, African-Americans, and Mexican-Americans with NIDDM in the U.S. Diabetes Care 1997;20:142-7.

10. Harris MI. Racial and ethnic differences in health care access and health outcomes for adults with type 2 diabetes. Diabetes Care 2001;24:454-9.

11. Miller CD, Barnes CS, Phillips LS, et al. Rapid A1c availability improves clinical decision-making in an urban primary care clinic. Diabetes Care 2003;26: $1158-63$.

12. Roethlisberger FS. Management and Morals. Cambridge (MA): Harvard University Press; 1941. 